\title{
DCE@urLAB: a dynamic contrast-enhanced MRI pharmacokinetic analysis tool for preclinical data
}

\author{
Juan E Ortuño ${ }^{1,2^{*}}$, María J Ledesma-Carbayo ${ }^{2,1}$, Rui V Simões ${ }^{3}$, Ana P Candiota ${ }^{1,4,5}$, Carles Arús $s^{4,1,5}$ \\ and Andrés Santos ${ }^{2,1}$
}

\begin{abstract}
Background: DCE@urLAB is a software application for analysis of dynamic contrast-enhanced magnetic resonance imaging data (DCE-MRI). The tool incorporates a friendly graphical user interface (GUI) to interactively select and analyze a region of interest $(\mathrm{ROI})$ within the image set, taking into account the tissue concentration of the contrast agent (CA) and its effect on pixel intensity.

Results: Pixel-wise model-based quantitative parameters are estimated by fitting DCE-MRI data to several pharmacokinetic models using the Levenberg-Marquardt algorithm (LMA). DCE@urLAB also includes the semi-quantitative parametric and heuristic analysis approaches commonly used in practice. This software application has been programmed in the Interactive Data Language (IDL) and tested both with publicly available simulated data and preclinical studies from tumor-bearing mouse brains.

Conclusions: A user-friendly solution for applying pharmacokinetic and non-quantitative analysis DCE-MRI in preclinical studies has been implemented and tested. The proposed tool has been specially designed for easy selection of multi-pixel ROls. A public release of DCE@urLAB, together with the open source code and sample datasets, is available at http://www.die.upm.es/im/archives/DCEurLAB/.
\end{abstract}

Keywords: DCE-MRI, Imaging, Levenberg-Marquardt, Fitting, Preclinical, Pharmacokinetics, Animal models, High field MR, IDL

\section{Background}

Dynamic contrast-enhanced magnetic resonance imaging (DCE-MRI) involves the acquisition of sequential images in rapid succession during and after the intravenous administration of a, usually, low-molecular weight contrast agent (CA), which includes a paramagnetic component such as gadolinium $\left(\mathrm{Gd}^{3+}\right)$. This functional imaging modality has proven to be useful in tumor differentiation, being a sensitive marker of antiangiogenic treatment effect $[1,2]$.

When $\mathrm{T}_{1}$-weighted magnetic resonance (MR) sequences are used, the CA induces a signal enhancement related

\footnotetext{
*Correspondence: juanen@die.upm.es

${ }^{1}$ CIBER de Bioingeniería, Biomateriales y Nanomedicina (CIBER-BBN), 50018 Zaragoza, Spain

2 Biomedical Image Technologies Group, Departamento de Ingeniería Electrónica, Universidad Politécnica de Madrid, 28040 Madrid, Spain

Full list of author information is available at the end of the article
}

with the shortening of spin-lattice or longitudinal relaxation time $\left(T_{1}\right)$, the time course of which can be related to physiological parameters. The most common CA used in $\mathrm{T}_{1}$-weighted DCE-MRI, Gadolinium-diethylenetriamine penta-acetic acid (Gd-DTPA), is able to transverse the vascular endothelium (except when the blood-brain barrier is intact) and enter the extravascular-extracellular space (EES), but is unable to cross the cellular membrane. Thus, in DCE-MRI the measured signal intensity changes derive mostly from CA that extravasates to the EES $[3,4]$. The dynamics of exchange between the capillary bed and the EES can be evaluated and are usually modeled as an open two-compartment model, dependent on the washout rate between EES and plasma $\left(k_{e p}\right)$, and the volume transfer constant between plasma and EES, denoted as $K^{\text {trans }}[5]$. 
DCE-MRI has been used to investigate permeability and perfusion in small animal tumor models [6,7]. A key consideration in rodents is that the concentration of $\mathrm{CA}$ in vascular plasma evolves rapidly compared to tissue, and is quite difficult to sample the maximum signal intensity to effectively characterize the tissue pharmacokinetics. Since sampling the blood (the gold standard in humans) is very invasive in small animals, kinetic models that do not rely on arterial input function (AIF) measurements are desirable in preclinical DCE-MRI.

Therefore, the software application presented in this manuscript is aimed at filing this gap and providing a powerful and versatile $\mathrm{T}_{1}$-weighted DCE-MRI processing tool, and at the same time, intuitive and easy-to-use in preclinical studies. It has been implemented in Interactive Data Language (IDL), accessible at http://www.exelisvis.com/ idl.

The DCE@urLAB application integrates pixel-wise pharmacokinetic analysis using the following models: Tofts [8], Hoffmann [9], Larsson [10], and a reference region (RR) model [11]. The Tofts pharmacokinetic model has been widely applied to characterize murine tumors [12-14], as well as the Hoffmann pharmacokinetic model $[15,16]$. The Larsson model has not been extensively applied to small animal DCE-MRI, but is the third model typically used in theoretical studies and reviews $[5,17]$. Finally, the RR model has been proposed as an alternative when AIF cannot be precisely estimated.

\section{Existing software}

Model-based and semi-quantitative analysis of $\mathrm{T}_{1}$ weighted DCE-MRI can be performed with general purpose pharmacokinetic compartmental analysis packages, either non-commercial, like WinSAAM [18], JPKD [19], or commercial, like SAAM II [20]. These are complex tools that require specific training and need to be adjusted to the particular problem of DCE-MRI. Pixel-wise analysis and ROI selection of images are also not included in these platforms.

Among the software specifically designed for DCE-MRI data are the packages BioMap [21], PermGUI and PCT [22], Toppcat [23], DcemriS4 [24], and DATforDCEMRI [25].

BioMap is built in IDL, and supports compartmental analysis over ROIs through the perfusion tool. Two ROIs must be defined, one describing the CA tissueconcentration and the other the concentration of the CA in blood plasma $\left(C_{p}\right)$. When $C_{p}$ cannot be measured in an ROI, either because the image does not contain a large blood vessel, or the signal from the blood vessel is corrupted by pulsation, movement or saturation effects, a theoretical bi-exponential decay function can be used as $C_{p}$. Published results with DCE-MRI using BioMap include small animal studies [12,26,27]. Although BioMap can generate pixel maps, it does not work with coarse resolutions and is limited to the Tofts model, with a bi-exponential model of $C_{p}$.

PermGUI and PCT [22] are freeware applications oriented to extract the permeability coefficient of the blood brain barrier (BBB) in human patients. The tools analyze DCE-MRI images using the Patlak model [28]. This model is also used in the package Toppcat, which runs as a plugin of ImageJ [29]. Toppcat is also free of charge for educational and research purposes.

DcemriS4 [24] is a collection of shell scripts to help automate the quantitative analysis of DCE-MRI and diffusion weighted imaging (DWI), and written in the $R$ programming environment [30]. Kinetic parametric estimation is performed with the Tofts model and nonlinear regression, Bayesian estimation or deconvolution algorithms. AIF is parameterized with a tri-exponential function [31] to obtain an analytical solution of the convolution integral and increase computational efficiency.

DATforDCEMRI [25] is an R package tool which allows performing kinetic deconvolution analysis [32] and visualizing the resulting pixel-wise parametric maps. Like DcemriS4, this software package requires an end-user training in $\mathrm{R}$ programming environment.

These software packages are primarily designed for human studies and thus are not well suited for some typical requirements of preclinical DCE-MRI, e.g., the difficulty in accurately measuring the AIF in small animals makes that typical models in human studies cannot be used and ultimately requires the use of the Hoffmann or RR models. These models are not implemented in available software packages. Other important functionalities such as the difficulty in reading the imaging format produced by preclinical studies prevent from the use of those packages by the preclinical research community. Thus, in-house solutions are commonly used in DCE-MRI small animal studies, using Matlab programming environment [33,34], LabView [35] or IDL [36-39], but they are mostly designed for a specific study and with limited availability.

\section{Implementation}

In this section, the compartmental models implemented in the DCE@urLAB analysis tool are described. Additional information and technical details can be found in the "DCEurLAB Methods.pdf" document included in the software package, accessible at http://www.die.upm.es/ $\mathrm{im} /$ archives/DCEurLAB/ and in the Additional file 1. This section also includes a brief description of the graphical user interface (GUI) usage.

\section{DCE-MRI pharmacokinetic modeling}

Model-based pharmacokinetic analysis of $\mathrm{T}_{1}$-weighted DCE-MRI used in the DCE@urLAB application tool is 
open bi-compartmental, representing the blood plasma and the EES, and assume some basic concepts in tracer kinetics and MR [5]. As the CA does not enter the intracellular space, this compartment is not considered in the model. The blood plasma is associated with the central compartment, the wash-out to the kidneys and the intake from the injected contrast, while the EES is the peripheral compartment. This compartmental scheme is shown in Figure 1. We should note that a bi-compartmental model does not consider the complex biology of the tumor. Although multi-compartment models have been proposed [40], the open bi-compartmental model has been able to fit DCE-DRI data surprisingly well and is therefore widely accepted by the research community. Time-course changes in tissue CA concentration are modeled as a result of first-order exchange of the CA molecules between compartments. A modified general rate equation [41] describes the CA accumulation and wash-out rate in the EES, under the assumption that the $\mathrm{CA}$ is well-mixed in the blood plasma:

$$
\frac{d C_{e}(t)}{d t}=\frac{K^{\text {trans }}}{v_{e}}\left(C_{p}(t)-C_{e}(t)\right), \quad v_{p} C_{p}+v_{e} C_{e}=C_{t}
$$

where $C_{e}$ is the CA concentration in EES, $C_{t}$ is the total CA concentration in the tissue, $v_{p}$ is the fractional volume of blood plasma, and $v_{e}=K^{\text {trans }} / k_{e p}$ is the fractional volume of EES. The physiological meaning of $K^{\text {trans }}$ depends on the biological mechanism of CA exchange (i.e., blood flow, permeability, or a mixed case). If no prior information about the tissue is available, then is prudent to leave the interpretation open.

\section{Tofts model}

The Tofts pharmacokinetic model $[8,42]$ is derived from Equation 1, excluding the contribution from vascular plasma. Tofts originally proposed a bi-exponential model

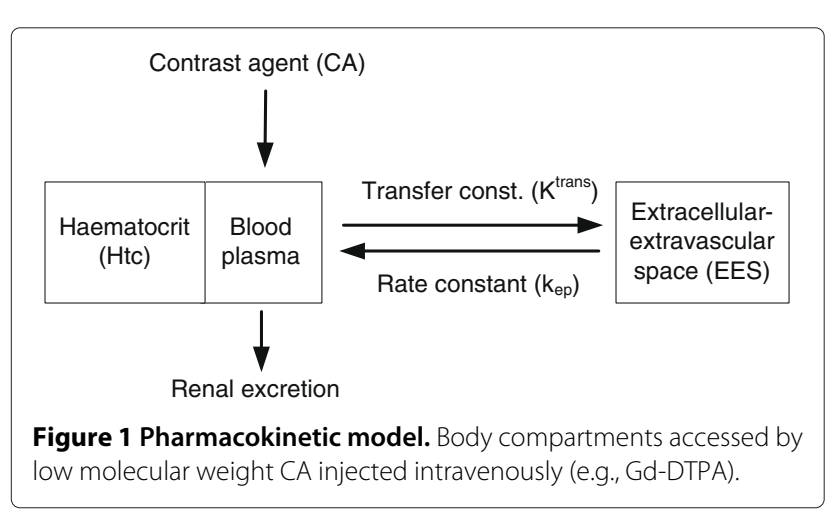

for $C_{p}$. In that case, the solution of Equation 1 for an instantaneous bolus injection reduces to:

$$
\begin{aligned}
& C_{t}(t)=D K^{\text {trans }} \sum_{i=0}^{2} a_{i} \frac{e^{-K^{\text {trans }} t / v_{e}}-e^{-m_{i} t}}{m_{i}-K^{\text {trans }} / v_{e}} \\
& C_{p}(t)=D \sum_{i=0}^{2} a_{i} e^{-m_{i} t}
\end{aligned}
$$

from which $K^{\text {trans }}$ and $v_{e}$ can be estimated through a minimization algorithm. Amplitudes $a_{i}$ and time constant $m_{i}$ are estimated from a population average and $D$ is the injected CA dose. The extended or modified Tofts model [5] corresponds to the adding of the contribution of the blood plasma fraction $v_{p} C_{p}(t)$ to account for the tracer in the vasculature. In this case, the unknown parameters are $K^{\text {trans }}, v_{e}$, and $v_{p}$. The discrete approximation measured, or population averaged vascular plasma CA concentrations at sampling times, can be solved with least-squares minimization methods, e.g., using the matrix-vector formulation of the discrete convolution:

$$
C_{t}(t)=C_{p}(t) *\left(K^{\text {trans }} e^{-k_{e p} t}\right)
$$

The Tofts model produces reliable results if the tissue is weakly vascularized, while the extended Tofts model can also be applied to highly perfused tumors [43]. It is important to note that the quantification of Tofts parameters requires the estimation $C_{p}(t)$ from the acquired MR signal. Thus, an additional MRI model has been included in the DCE@urLAB application and is discussed later.

\section{Hoffmann model}

The Hoffmann model [9] is derived from the Brix model [44] for fast bolus injection, and assumes that the CA transfer from blood plasma to EES is a slow process. The model establishes a direct relationship between MR signal enhancement and CA exchange rates, without the need for AIF estimation and MR quantification. After the bolus injection, the model is described as:

$$
\frac{S(t)}{S_{0}}=1+A^{H} k_{e p}\left(\frac{e^{-k_{e p} t}-e^{-k_{e l} t}}{k_{e l}-k_{e p}}\right)
$$

where $S(t)$ is the MR signal course from tissue and $S_{0}$ is the MR signal before CA injection. The fitting parameters are: $k_{e p} ; A^{H}$, which approximately corresponds to the size of the EES; and $k_{e l}$, the renal elimination constant.

\section{Larsson model}

The Larsson model [10] uses a known blood plasma CA concentration course, either measured from blood samples or estimated from the MRI data. It is assumed that 
the MR signal is linearly related to the CA concentration. In that case, the MR signal is modeled as:

$$
S(t)=S_{0}+\left(\frac{\dot{S}(t)}{\sum_{i=0}^{N} a_{i}}\right) \sum_{i=0}^{N} a_{i} \frac{e^{-k_{e p} t}-e^{-m_{i} t}}{m_{i}-k_{e p}}
$$

where $\dot{S}(t)$ is the initial slope of the MR signal and $S_{0}$ the MR signal value prior to CA injection. $C_{p}$ is approximated as a sum of $\mathrm{N}$ exponentials with amplitudes $a_{i}$ and time constant $m_{i}$.

\section{$R R$ model}

An alternative to a populations-based or estimated AIF, is the RR model [11]. The approach uses a well-characterized tissue to combine two versions of Equation 1, one for the RR and another one for the tissue of interest. This allows the removal of $C_{p}$ in the solution of the resulting equation [11].

$$
\begin{aligned}
C_{t}(t)= & \frac{K^{\text {trans }}}{K^{\text {trans }, r}}\left(C_{t, r}(t)+\left(\frac{K^{\text {trans }, r}}{v_{e, r}}-\frac{K^{\text {trans }}}{v_{e}}\right)\right. \\
& \left.\times \int_{0}^{t} C_{t, r}\left(t^{\prime}\right) e^{-K^{\text {trans }}\left(t-t^{\prime}\right) / v_{e}} d t^{\prime}\right)
\end{aligned}
$$

where $C_{t, r}$ is the concentration of CA in the RR tissue and $K^{\text {trans,r } r}$ and $v_{e, r}$ are the quantitative parameters for the RR.

\section{MRI model}

The Tofts and RR models require the calibration of CA concentration from measured MRI parameters. If the bulk magnetic susceptibility (BMS) shift is negligible, the relationship between $\mathrm{T}_{1}$ and CA concentration is determined by the Solomon-Bloembergen equation [45]:

$$
\frac{1}{T_{1}(t)}=\frac{1}{T_{10}}+r_{1} C_{t}(t)
$$

where $T_{10}$ is the $T_{1}$ value before CA injection and $r_{1}$ is the longitudinal relaxivity. The relationship between $\mathrm{CA}$ concentration and the relative increase in signal intensity can be derived from the Bloch equations for any imaging sequence, e.g., the signal for a $\mathrm{T}_{1}$-weighted spin-echo pulse sequence (at short echo time) with repetition time (TR) is:

$$
S(t)=S_{0}\left(1-e^{T R / T_{1}(t)}\right)
$$

From Equation 7 and $8, C_{t}$ is equal to:

$$
C_{t}(t)=\frac{1}{r_{1}}\left(\frac{1}{T R} \ln \left(\frac{S_{0}}{S_{0}-S(t)\left(1-e^{-T R / T_{10}}\right)}\right)-\frac{1}{T_{10}}\right)
$$

For spoiled gradient-echo pulse sequences with flip angle $\alpha$, the MR signal is equal to:

$$
S(t)=\left(\frac{S_{0}\left(1-e^{-T R / T_{1}(t)}\right) \sin \alpha}{1-e^{-T R / T_{1}(t)} \cos \alpha}\right)
$$

The signal intensity is converted to CA concentration in tissue using the equation from [46] to calculate $T_{1}(t)$ :

$$
\begin{aligned}
T_{1}(t)^{-1} & =-\frac{1}{T R} \ln \left(\frac{1-\left(\frac{S(t)-S_{0}}{S_{0} \sin \alpha}+\frac{1-m}{1-m \cos \alpha}\right)}{1-\left(\frac{S(t)-S_{0}}{S_{0} \sin \alpha}+\frac{1-m}{1-m \cos \alpha}\right) \cos \alpha}\right), \\
m & =e^{-T R / T_{10}}
\end{aligned}
$$

and CA concentration in tissue is calculated from Equation 7. Note that $r_{1}$ and $\mathrm{T}_{10}$ must be known to quantify the tissue concentration from the MR signal. $\mathrm{T}_{10}$ may be estimated using the ratio of two spin-echo images collected with different TR. The estimation error can be reduced with a higher number of images with a least-squares minimization algorithm.

\section{Estimation of model parameters}

Curve fitting routines have been implemented using internal IDL functions and the freely available MPFIT IDL library [47]. MPFIT contains a set of non-linear regression algorithms for robust least-squares minimization, based on the freely available MINPACK package (Univ. of Chicago, http://www.netlib.org/minpack/) a library of FORTRAN subroutines for solving nonlinear equation systems.

DCE@urLAB uses the Levenberg-Marquardt algorithm (LMA) [48] to perform the non-linear least squares regression in each pixel of the analyzed ROI. LMA has demonstrated robustness in the pharmacokinetic modeling of DCE-MRI [49]. LMA is used to estimate appropriate parameters in several models: Tofts (with bi-exponential $C_{p}$ of Equation 2 or solving the discrete convolution Equation 3); the equivalent extended Tofts model; Hoffmann (Equation 4); Larsson (Equation 5); and also the RR model (Equation 6).

Pixel-based processing of dynamic MRI data can be demanding in terms of memory and CPU, and hardware requirements will vary depending on the size of data sets, as well as the number of pixels selected. In any case, it is recommended to run the program in systems with at least $2 \mathrm{~GB}$ of RAM memory. In addition to pharmacokinetic modeling, model-free semi-quantitative analysis can be performed, including IAUC (initial area under curve), RCE (relative contrast enhancement) and TTM (time to max enhancement) [50]. 


\section{Description and use of the GUI}

The DCE@urLAB GUI is composed of a main window, which opens when the tool is executed, and auxiliary windows for results, input/output processes, or auxiliary activities. Figure 2 shows an appearance of the main window once the DCE-MRI study is loaded in memory. The complete and detailed functionality of the GUI is described in the user manual included in the downloadable software package. A general overview is presented in this section.

\section{Input data}

The software tool accepts DCE-MRI sequences and auxiliary inputs: $\mathrm{T}_{10}$ maps, AIF data and pre-calculated ROIs. Interface functionality is disabled until a 4dimensional DCE-MRI study is open. The tool considers the sequence set to be a $4 \mathrm{D}$ stack of images in $\mathrm{X}-\mathrm{Y}-\mathrm{Z}$ time order. Data can be imported from DICOM format, Bruker Biospin MRI data format (http://www.bruker.com/ products $/ \mathrm{mr} / \mathrm{mri}$.html), as well as from binary unformatted data. If the dynamic MR sequence is loaded properly, the interface will show a single $2 \mathrm{D}$ slice of the whole
4D data set in the left display tab, and a relative contrast enhancement (RCE) image in the right display tab (Figure 2).

The platform is specially designed to perform ROI or pixel-wise analysis over the selected ROI belonging to a single slice in the $\mathrm{Z}$ dimension (Z-slice for short). These ROIs can be exported in a custom format and subsequently imported in another work session. When required for a specific MRI model, $\mathrm{T}_{10}$ maps can be loaded from the menu file tab. AIF data can also be imported from previously saved sessions or external acquisitions.

\section{Displaying data sets}

After loading a valid DCE-MRI sequence, main processing options and menus will become activated. The user is now able to select ROIs, change parameters, as well as configure visualization options. Nevertheless, other options will not be activated until a valid ROI is drawn or imported.

The user can navigate through dynamic frames or Zslices to select an active ROI for the pharmacokinetic analysis. The color palette of both MRI and RCE displays can be changed by selecting this option on the menu

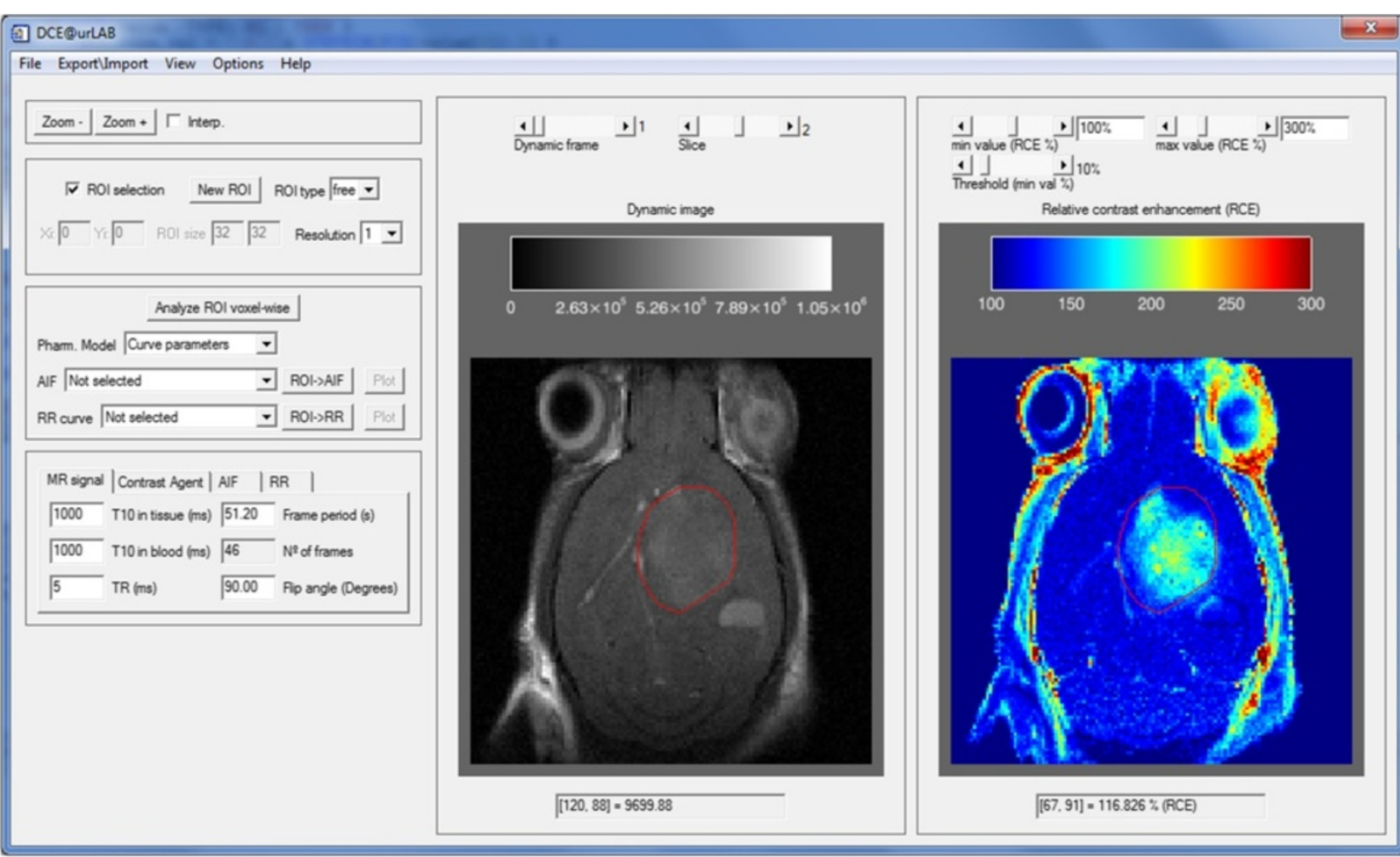

Figure 2 Main window interface. In this example, a mouse brain tumor study is displayed: GL261 glioblastoma (see also the Results section). The main window interface is shown before the ROI has been selected. In the right side of the window, the RCE image is drawn with a "rainbow" color palette. The DCE-MRI image is drawn with "black \& white" color palette in the central part. The Upper slides allow changing the current time frame and Z-slice. Other tabs, such as zoom options, ROI selection options, parametric selection and initial constants, are grouped in the left side of the main window interface. 
bar (options drop down menu). The user can additionally change the brightness, contrast, alpha channel, etc. Pressing mouse buttons on the display images produces different actions depending on the ROI selection mode. When the ROI selection mode is not activated, the actions allowed are:

- Pressing the right mouse button on any image will plot the dynamic MR signal course of the pointed pixel.

- If the left mouse button is pressed over the MRI window, the value of the current pixel appears in the information label located at the bottom of the MRI window tab.

- When the left mouse button is pressed on the RCE image, the RCE value (\%) of the current pixel will be shown in the associated information label.

\section{Selecting and defining ROIs}

If the ROI selection mode is activated, right and left mouse buttons are used to manually place ROIs in the selected slice. The ROI types can be Box, Full or Free-drawing type. The ROI definition depends on the type of ROI selected. If a Box-type is selected, the upper left and bottom right corners of the ROI are defined by pressing the left mouse button over the image, or alternatively, typing their $\mathrm{X}$ and $\mathrm{Y}$ coordinates in editable text fields. If the Full ROI type is selected, the current Z-slice is then defined as a ROI. In the Free-drawing ROI type, the user moves the pointer while pressing down the left mouse button over the image to manually delineate the contour of the ROI. The ROI can be deleted in every moment using the New ROI button and starting again. Finally, the user must also choose the resolution in the $\mathrm{Z}$-slice, i.e., select the pixel size for processing options. The finest resolution corresponds to the intrinsic resolution of the image, but the user can also select coarser resolutions from $2 \times 2$ to $10 \times 10$ pixels in the Z-slice ( $x$-y plane). This option allows a direct comparison with other applications using low-resolution maps. The selected ROIs are currently limited to a single Z-slice.

\section{Input parameters}

Processing input parameters should be checked before each ROI analysis to obtain accurate results. Input parameters are organized in tabs (located on the lower-right of the main window interface). Each tab groups a set of related parameters. The MR signal tab contains MRI data related constants (e.g., frame period, repetition time, etc.). The AIF tab groups the parameters used in the bi-exponential model for the CA concentration in blood plasma proposed by Tofts. The CA tab must be completed with information concerning the injected contrast (e.g., injection frame, relaxivity, injected dose, etc.). Finally, the RR tab contains additional data used in the reference region model. These input parameters will be used or not depending on the pharmacokinetic study selected, e.g., the AIF tab is only read when the Tofts model is applied.

\section{Pharmacokinetic processing and analysis}

Pharmacokinetic models are estimated by pressing the Analyze ROI button. Note that this option is inactive until
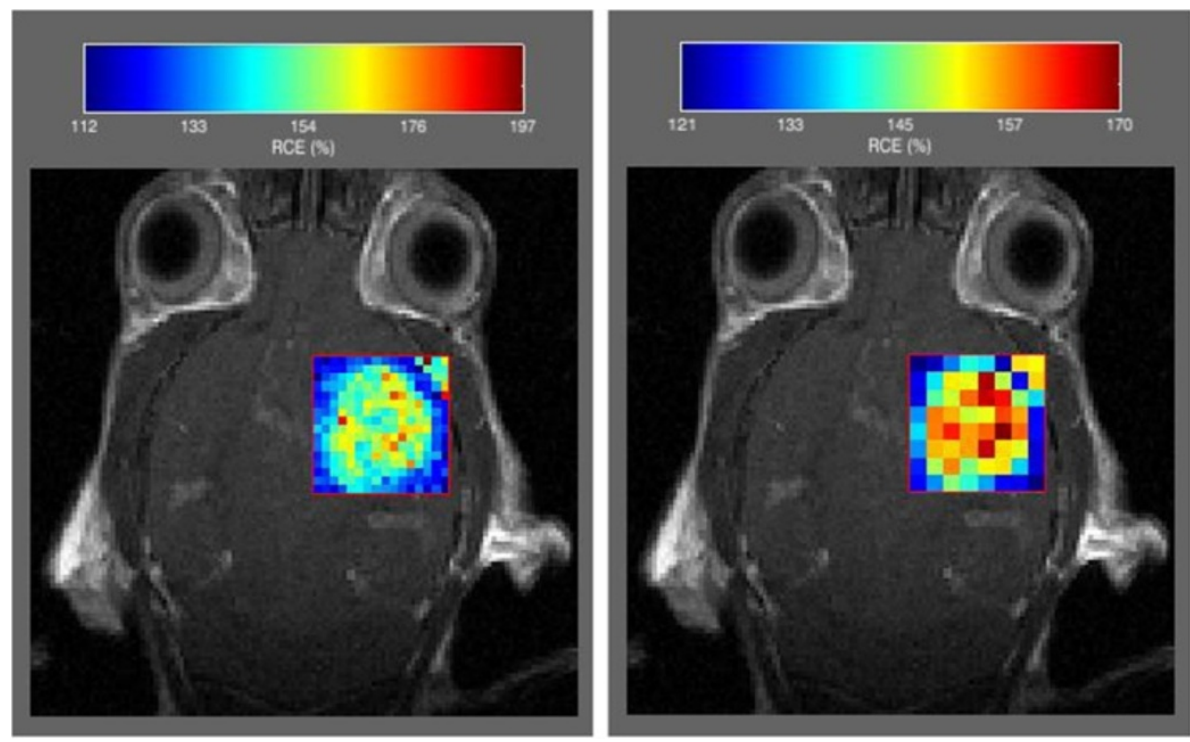

Figure 3 Pixel resolution. The pixel where pharmacokinetic modeling is performed can vary in resolution: From intrinsic image resolution (the finest) to coarse resolution. In the figure, two different coarse resolutions are shown for a mouse GL261 glioblastoma. 

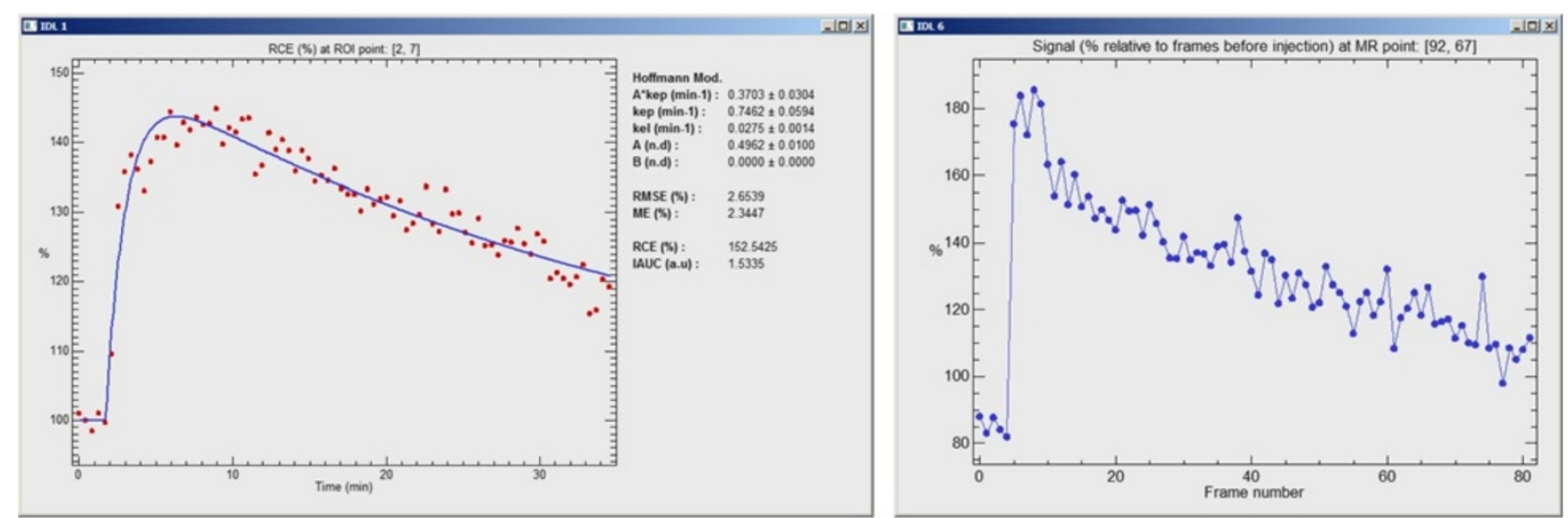

Figure 4 Modeled and acquired DCE-MRI curves. Modeled (left) and acquired (right) DCE-MRI curves. The software tool can plot the time-course changes in individual pixels or in the whole ROI. The curve can be compared with the analytic pharmacokinetic model (left plot), where the acquired data are represented as dots and the fitted evolution as a continuous curve.

a valid ROI has been previously drawn or imported. Hoffmann, Tofts (standard and extended), Larsson and RR models can be selected for analysis. Model-free parameters (i.e., semi-quantitative parameters) are included as an independent option. Analytical or numerical solutions of the convolution integral are automatically chosen depending on the type of AIF loaded. Once the analysis is finished, the user can select the parameter to be displayed or saved in disk, by using the drop lists associated to each pharmacokinetic model. An example of the result with Box-type ROIs and two different resolutions is shown in Figure 3. The visualization menu located on the

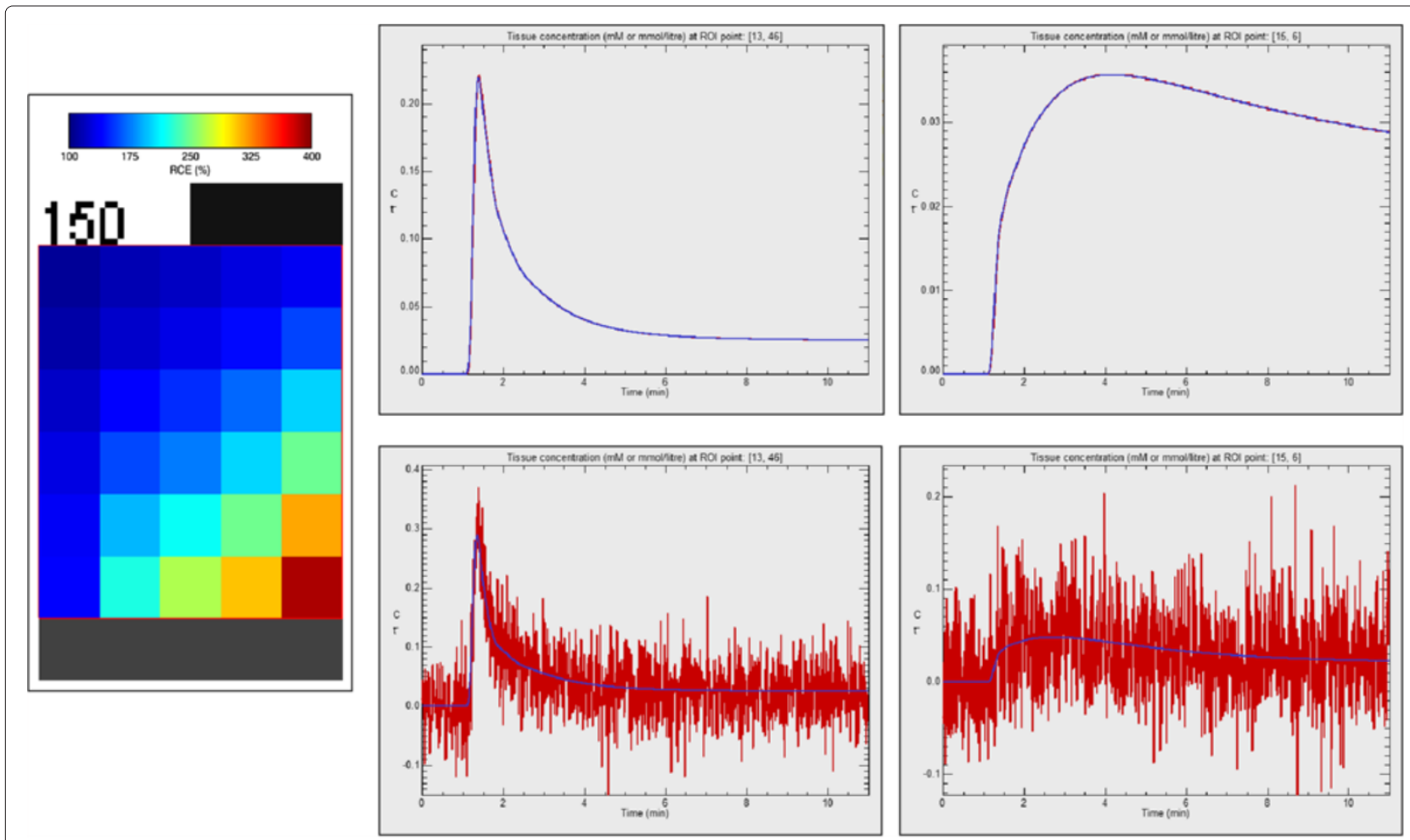

Figure 5 QIBA test data corresponding to the Tofts model. Left: RCE values of 30 combinations of $K^{\text {trans }}$ and $v_{e}$ values of simulated QIBA test data without added noise. Upper-right: curve-fitting with the Tofts model over two random points of the QIBA test data without noise. Lower-right: curve-fitting of the Tofts model adding Gaussian noise of zero mean and $\sigma=20 \%$ of the signal baseline. 

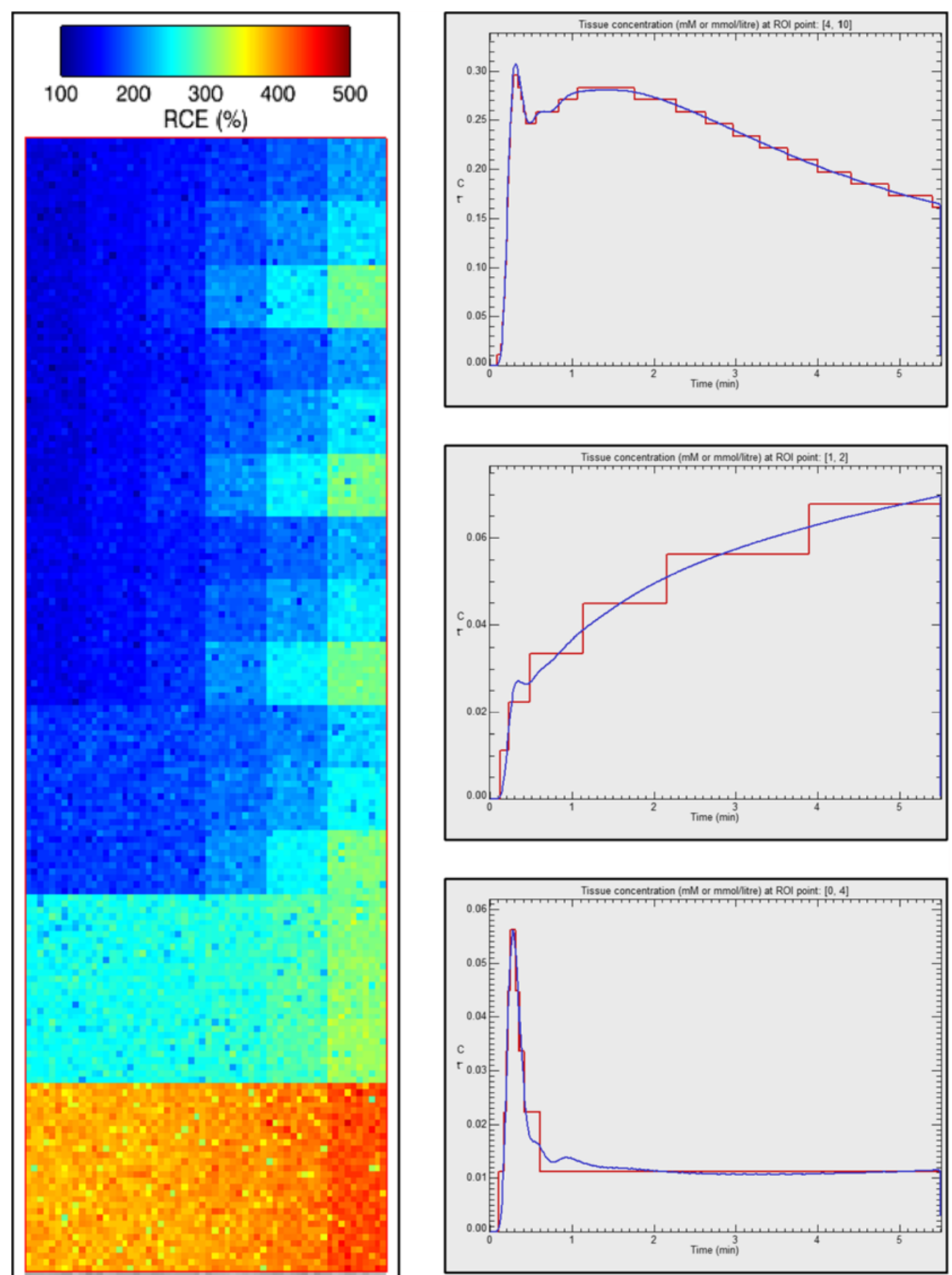

Figure 6 QIBA test data corresponding to the extended Tofts model. Left: RCE values of 108 parameter combinations, adding Gaussian noise of zero mean and $\sigma=20 \%$ of signal prior to CA injection. Right: Extended Tofts model fitting over three random ROls where each ROI comprises a single $10 \times 10$ pixels box with common parameters. 
left (Figure 2) can select the transparency and scale of the parametric map.

The software tool also provides detailed information of the estimated pharmacokinetic model at pixel level; if the left mouse button is pressed when the pointer is located over the ROI, the adjusted curve of the parametric model associated to the selected pixel is plotted together with the DCE-MRI sequence values. An example of this plot is shown in Figure 4. The plot represents the model curve with the estimated parameters displayed on the right side.

Complementary results and data can be accessed from the menu bar, e.g., in the Export/import drop-down menu, several options can be selected to export images shown on the screen, ROI kinetics, or the set of parametric values of the selected ROI. Single column, multiple column, and matrix format are available.

\section{Results}

\section{Validation using simulated data}

Tofts and extended Tofts models have been validated with the Quantitative Imaging Biomarkers Alliance (QIBA) DCE-MRI synthetic data, which are publicly available at http://dblab.duhs.duke.edu. The physiologic model is described in [51] and was simulated using JSIM [52]. Two sets of DCE-MRI images were used, corresponding to the Tofts model and the extended Tofts model. Data is available in DICOM part 10 format. Simulation parameters of the Tofts model were: Flip angle, $30^{\circ}$; TR, 5 ms; time interval between frames, $0.5 \mathrm{~s} ; \mathrm{T}_{10}$ in tissue, $1000 \mathrm{~ms}$; $\mathrm{T}_{10}$ in blood vessels, $1440 \mathrm{~ms}$; Haematocrit, $45 \%$. A 10 minute study was simulated, with injection of CA occurring at $60 \mathrm{~s}$. The data in the test images was generated using several combinations of $K^{\text {trans }}$ and $v_{e} . K^{\text {trans }}$ takes values $\{0.01,0.02,0.05,0.1,0.2,0.35\} \mathrm{min}^{-1}$ and $v_{e}$ takes $\{0.01$,

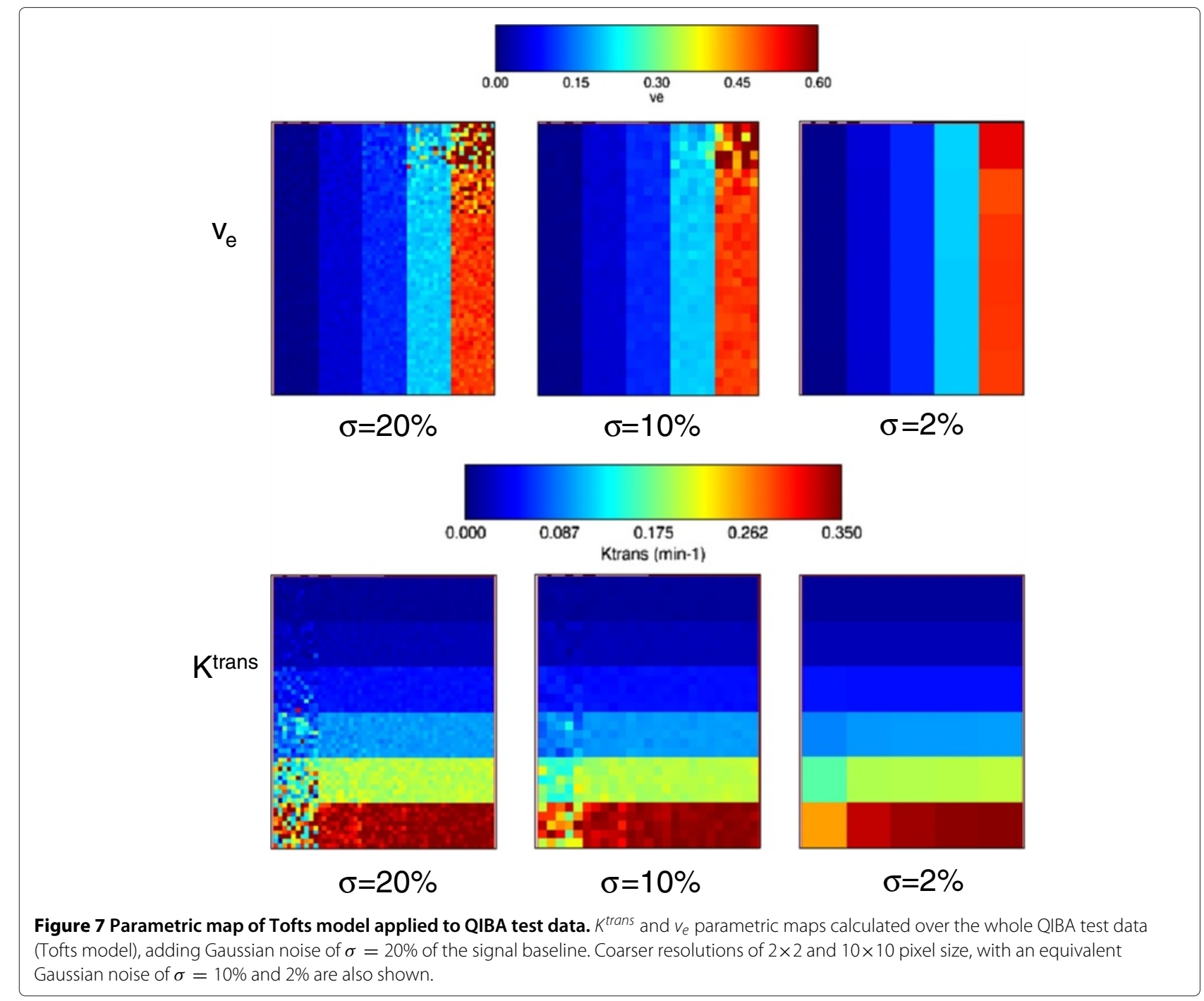




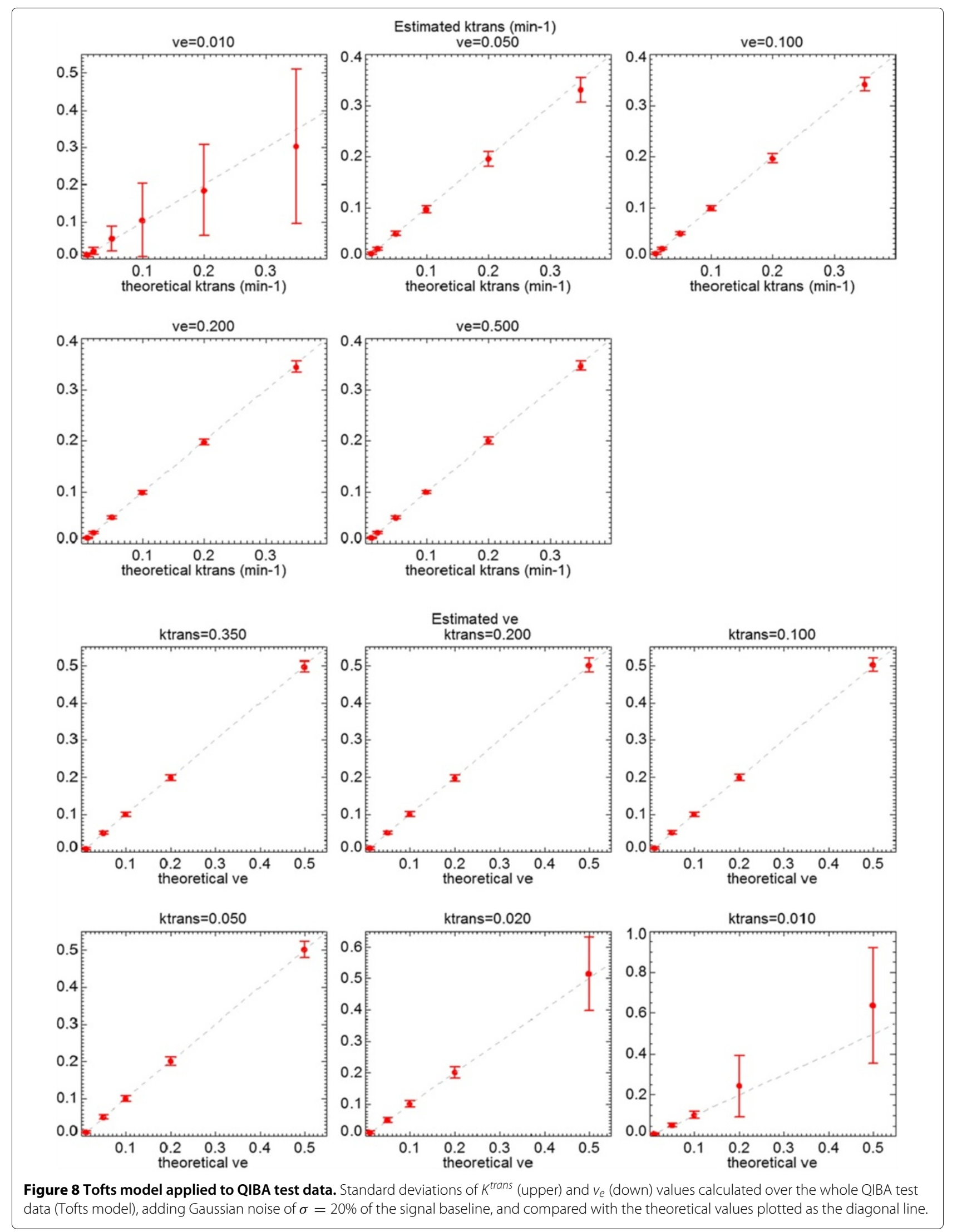


$0.05,0.1,0.2,0.5\}$. The image frames contain $10 \times 10$ pixels patches of each and combination. The vascular region was located in the bottom strip of the image. An RCE image is shown in Figure 5.

The extended Tofts model data have the following parameters: Flip angle, $25^{\circ}$; TR, $5 \mathrm{~ms}$; time interval between frames, $0.5 \mathrm{~s}$; $\mathrm{T}_{10}$ in tissue, $1000 \mathrm{~ms}$; $\mathrm{T}_{10}$ in blood vessels, $1440 \mathrm{~ms}$; Haematocrit, 45\%. A 3.5 min study is simulated, with injection of CA occurring at $5 \mathrm{~s}$. The data were generated using combinations of $K^{\text {trans }}, v_{e}$ and $v_{p}$; $K^{\text {trans }}$ varies over $\{0,0.01,0.02,0.05,0.1,0.2\} \mathrm{min}^{-1}, v_{e}$ takes values $\{0.1,0.2,0.5\}$, while $v_{p}$ takes $\{0.001,0.005$, $0.01,0.02,0.05,0.1\}$. Each combination of these three parameters is contained in a $10 \times 10$ pixel patch. The vascular region is the bottom $60 \times 20$ pixels strip of the image. An RCE image of this test data is represented in Figure 6. The kinetic variation of three different combinations of parameters is also shown in Figure 6. It can be appreciated that the discretization uncertainty in this data set is larger than in the former data set (Figure 5), and it is due to the lower value of equilibrium magnetization used in the simulation.

\section{Results with Tofts model applied to QIBA test data}

Gaussian noise of zero mean and standard deviation $(\sigma)$ equal to $20 \%$ of the signal baseline was added to the test data set. Box-type ROI covering the whole tissue region was selected (i.e., $50 \times 60$ pixels with 30 combinations $K^{\text {trans }}$ and $v_{e}$ values). Coarser resolutions were also studied (i.e., $2 \times 2$ and $10 \times 10$ pixel size), with an equivalent Gaussian noise of $\sigma=10 \%$ and $2 \%$ of the signal baseline, respectively. Noise level of $\sigma=20 \%$ is appreciated in lower-right images in Figure 5, compared with noise free dynamic values of the same two pixels, shown in the upper-right graphs. The fitting of discrete convolution of Equation 3 was applied to all pixels in the selected ROI. Graphical results for and values are represented in Figure 7. Standard deviations referenced to the theoretical values are represented in Figure 8 for $K^{\text {trans }}$ (up) and $v_{e}$ (bottom).

\section{Results with Extended Tofts model applied to QIBA test data}

Gaussian noise of zero mean and $\sigma=20 \%$ of the signal baseline was added to the test data set. A Box-type ROI of $60 \times 180$ pixels was selected to cover the 108 combinations of $K^{\text {trans }}, v_{e}$ and $v_{p}$ values. A coarser resolution map of $5 \times 5$ pixel size, which reduces noise $\sigma=4 \%$ of the baseline signal level, was also calculated. Color maps of the resultant parameters are represented in Figure 9. Standard deviations and bias referenced to the theoretical values are represented in Figure 10 for $K^{\text {trans }}$ (up) and $v_{p}$ (bottom). $v_{e}=0.5$ was used in all cases.

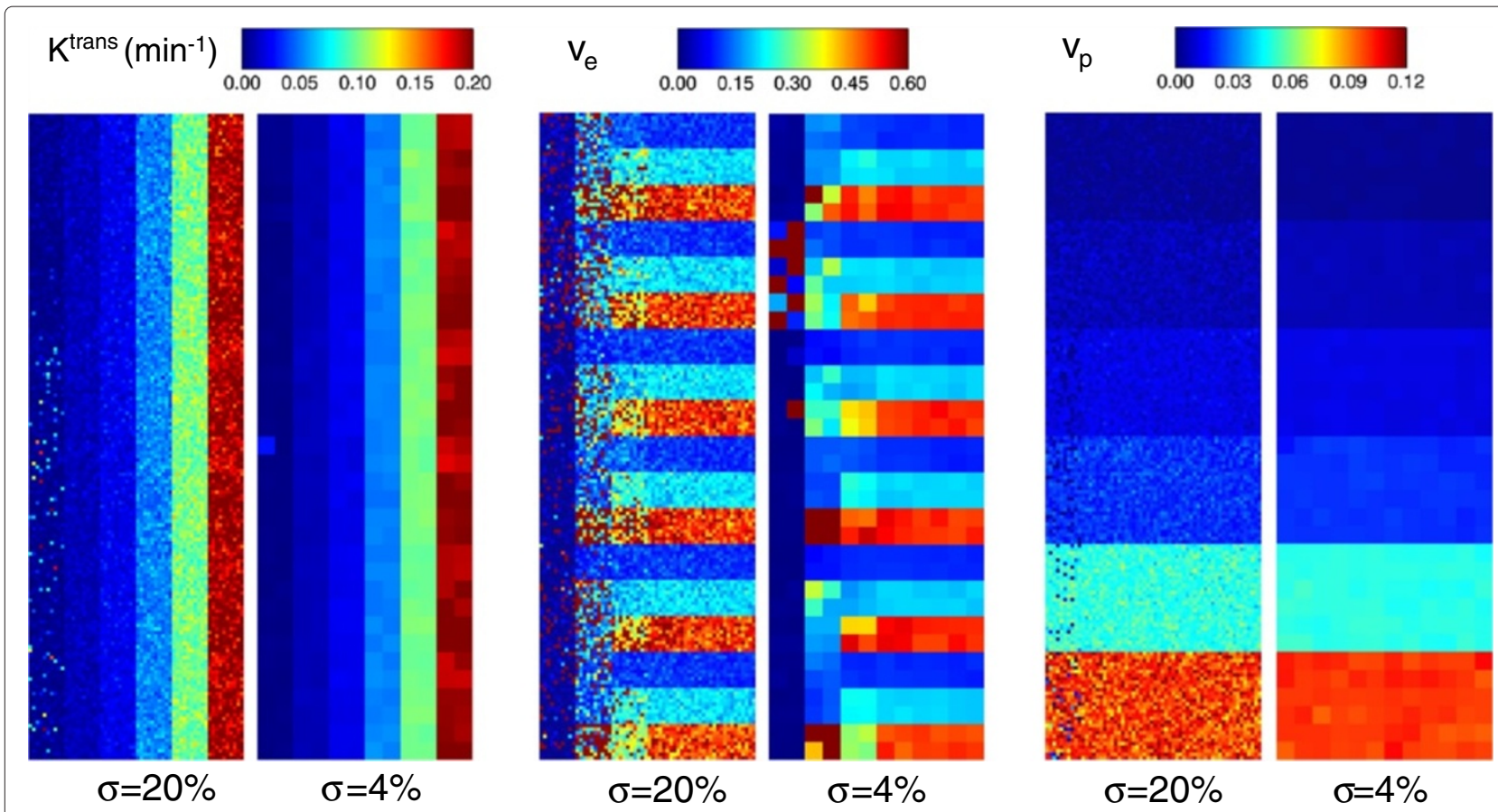

Figure 9 Parametric map of extended Tofts model applied to QIBA test data. Values of $K^{\text {trans }}, v_{e}$ and $v_{p}$ maps using the extended Tofts model supplied in the QIBA test data, adding Gaussian noise of $\sigma=20 \%$ of the signal baseline. Parametric maps using resolution of $5 \times 5$ pixels, resulting in an equivalent noise of $\sigma=4 \%$, are also shown. 


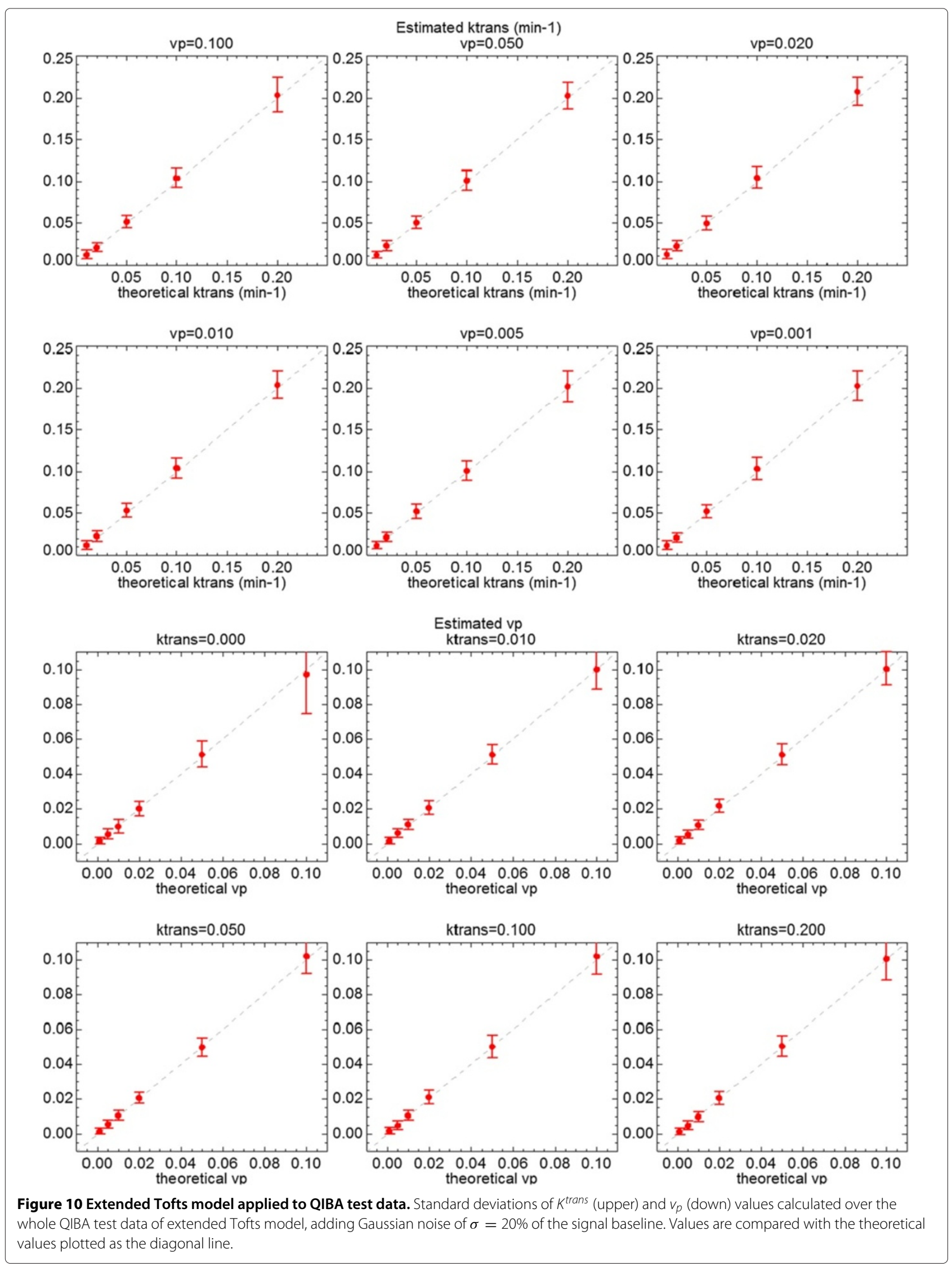




\section{Example with mouse brain tumor}

The platform has been tested over real acquisitions of $\mathrm{T}_{1}$-weighted DCE-MRI small animal data. Two different C57BL/6 mouse models have been used in this study. First, a genetically engineered mouse (GEM, S100-v-ErbB; Ink4a-Arf(+/-)), female, age 40 weeks, bearing a Schwannoma (confirmed by histopathological studies carried out by Dr. Martí Pumarola, Murine Pathology Unit, Centre de Biotecnologia Animal i Teràpia Gènica, $U A B$ ). Animals from this colony generally develop oligodendrogliomas [53], although a small percentage of animals can develop other tumour types [54]. The second model studied was a mouse bearing a stereotactically-induced GL261 glioblastoma, described elsewhere [55,56], age 20 weeks.

A bolus of CA (Gd-DTPA -Magnevist, Bayer Schering Pharma AG, Berlin, Germany-, $50 \mathrm{mM}$ in saline, $0.2 \mathrm{mmol} / \mathrm{kg}, 10 \mathrm{~s}$ duration) was manually injected after acquiring five pre-contrast images. A series of 41 dynamic spin-echo images was acquired with temporal resolution of $51.2 \mathrm{~s}$ per frame and the following parameters: TR/TE, $200 / 5 \mathrm{~ms}$; field of view, $17.6 \times 17.6 \mathrm{~mm}^{2}$; slice thickness, $1 \mathrm{~mm}$; in-plane resolution, $138 \times 138 \mu \mathrm{m} /$ pixel. The studies were carried out at the joint NMR facility of the Universitat Autònoma de Barcelona and CIBER-BBN (Cerdanyola del Vallès, Spain), using a 7 T horizontal magnet (BioSpec 70/30; Bruker BioSpin, Ettlingen, Germany).

Pixel-wise Hoffmann analyses were performed over a manually delineated ROI in a Z-slice for both cases (Figures 11 and 12, top). The MR signal courses are shown in Figures 11 and 12 (bottom) with significant differences in their biophysical parameters. For the GEM Schwannoma case, $k_{e p}$ estimated values were $0.54 \pm 0.05 \mathrm{~min}^{-1}$ for pixel (1) and $0.03 \pm 0.01 \mathrm{~min}^{-1}$ for pixel (2), while pixel (3) region contains highly vascularised tissue and the Hoffmann model did not apply correctly in these cases (only a few pixels presented acceptable fittings: an example is shown in Figure 11, estimated $\left.k_{e p}=2.81 \pm 0.46 \mathrm{~min}^{-1}\right)$. For the GL261 glioblastoma example (Figure 12), the $k_{e p}$ estimated values were $1.41 \pm 0.23 \mathrm{~min}^{-1}$ (tumour border, better perfusion) and $0.29 \pm 0.01 \mathrm{~min}^{-1}$ (tumour core). The mean $k_{e p}$ value for this tumour was $0.77 \pm 0.35 \mathrm{~min}^{-1}$, which is similar to

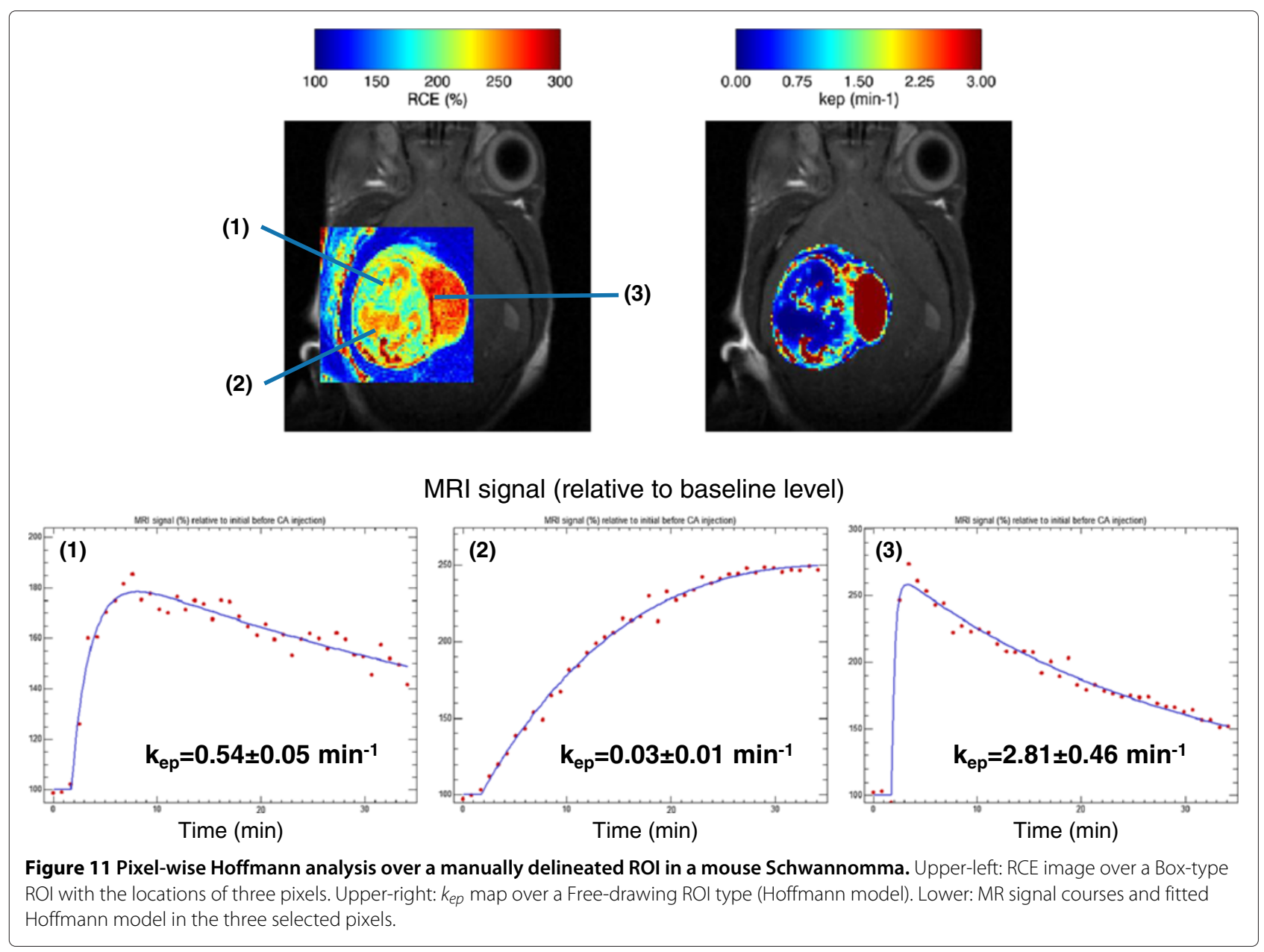




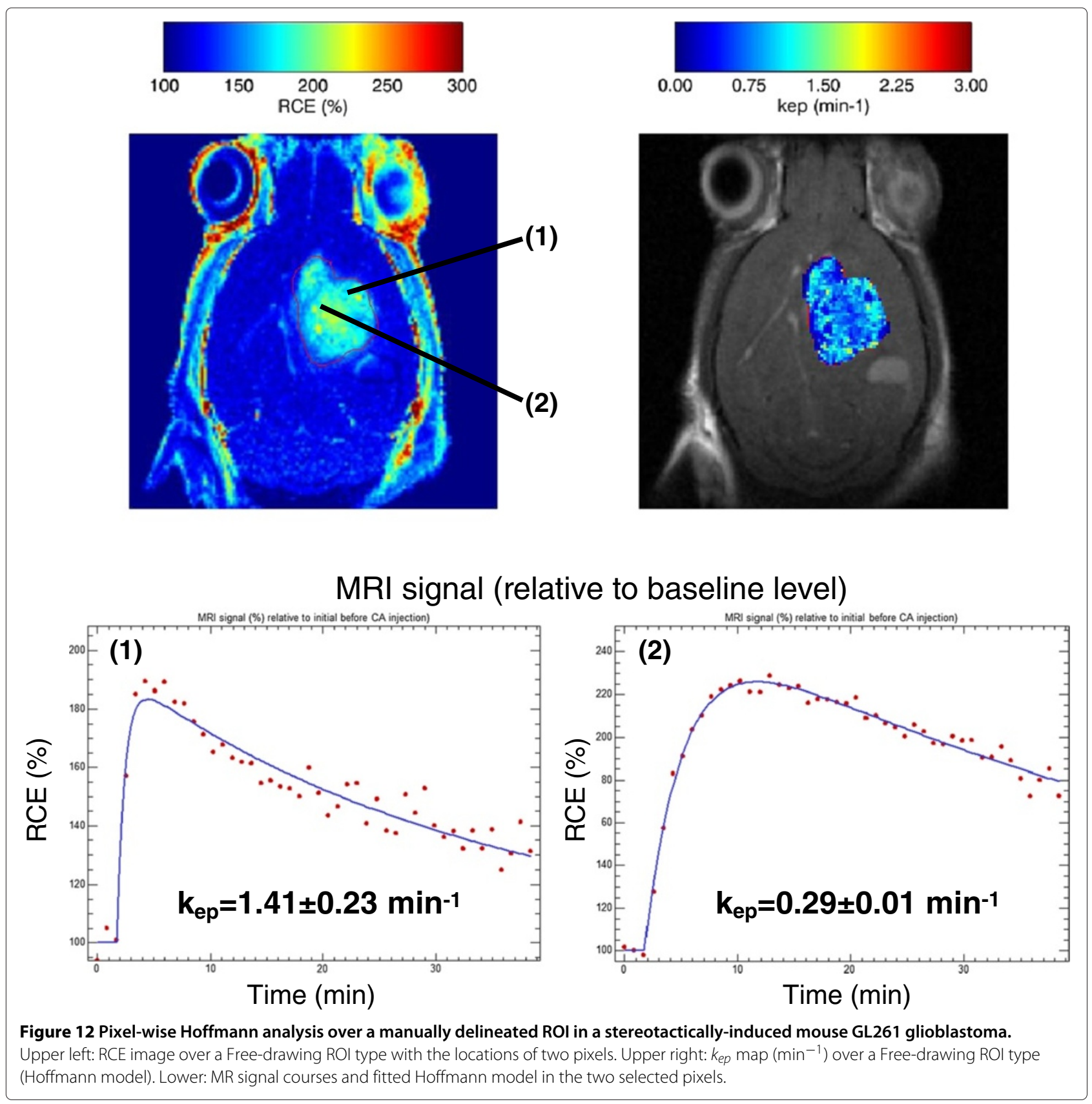

the mean values calculated for other GL261 cases in our group $(0.87 \pm 0.59, \mathrm{n}=8)$. These values also agree with previously described studies in the literature. For example, it was possible to calculate $k_{e p}$ from $K^{\text {trans }}$ and $v_{e}$ values reported by authors in [46], which studied a rat glioma model: the $k_{e p}$ value calculated was $0.86 \mathrm{~min}^{-1}$. Regarding to mouse glioma models, the same $k_{e p}$ estimation approach was possible from the study performed in [7] taking into account graphs in their page 612: the estimated $k_{e p}$ value in this case was $0.75 \mathrm{~min}^{-1}$, for tumours with a volume $\left(60-80 \mathrm{~mm}^{3}\right)$ similar to our GL261 $\left(69 \pm 43 \mathrm{~mm}^{3}\right)$.
In both cases, the differences observed in the MR signal time courses between well-perfused and badly perfused (hypoxic regions) agree with the ones described by authors in $[15,57]$.

\section{Computational implementation and requirements}

DCE@urLAB has been implemented in a flexible and modular way, so that the addition of new analysis models is straightforward. The different models can also be used as inline functions to allow flexibility of use and batch programming of multiple studies for advanced users. 
Regarding complexity, the optimization (LMA) performed in each pixel has a global algorithmic complexity bound dependent on stopping criterion and number of maximum iterations. The algorithmic complexity by iteration is determined by the cost function (i.e., the pharmacokinetic model) through the calculation of its jacobian matrix. It has been experimentally verified that the computing time needed to perform a pharmacokinetic analysis depends linearly on the number of pixels contained in the ROI and the number of dynamic frames of DCE dataset. This behaviour is expected since the average number of iterations of the LMA does not substantially change for large number of pixels. For example, in a $2.8 \mathrm{GHz}$ Intel Quad Core CPU with 8 GB RAM personal computer, it took 20 seconds to fit a ROI of 1024 pixels and 40 dynamic frames to the Tofts model. Although unrealistic, because tumor ROIs are smaller, the complete analysis using the Tofts model of the whole DCE dynamic slice $(128 \times 128=16384$ pixels $)$ and 40 dynamic frames, took about 5 minutes in the personal computer formerly described. A maximum of 1.5 GB RAM was required in this case. Should more computer power be required (e.g., with higher resolution images), the program could be easily parallelized and several cores used.

DCE@urLAB is designed to run under Microsoft Windows XP/Vista/7 (both 32 and 64 bits). In order to use the application tool, IDL (version 6.4 or posterior) must have been installed. Another possibility is to install the IDL virtual machine (version 6.4 or posterior), which can be downloaded freely and does not require a license.

\section{Conclusions}

Up to date there is no friendly software application for pixel-wise and ROI analysis of DCE-MRI data that can apply different pharmacokinetic models in a preclinical environment. DCE@urLAB is a user-friendly software designed to fulfill the potential needs of the preclinical DCE-MRI community. It has been focused on the analysis of $\mathrm{T}_{1}$-weighted DCE-MRI studies, and tested and optimized according to the requirements of preclinical data analysis. The proposed tool has also been specially designed for easy selection of multi-pixel ROIs. The platform incorporates the compartmental pharmacokinetic models of Tofts, Hoffmann, Larsson, and RR, complemented with non-parametric analysis. Pixel-wise and ROI options allow the user to choose from a variety of forms and pixel sizes (i.e., resolutions). If required by the model, AIF and $\mathrm{T}_{10}$ maps can also be estimated from the acquired data. DCE@urLAB reads multi-slice DCE-MRI data from proprietary and binary raw formats. Results can be exported as color maps superimposed to the DCE image, or as text files that can easily be read with other statistical software packages. Individual pixel and ROI dynamic curves can also be visualized, for easy expert interpretation and pharmacokinetics validation. The most relevant and used models in literature (Tofts models) have been validated with publicly available simulated data. Preliminary experiments have been conducted using $\mathrm{T}_{1}$-weighted DCE-MRI dynamic data from tumor-bearing mouse brains. A public release of DCE@urLAB, together with the open source code and sample datasets, is available at http://www.die.upm.es/im/ archives/DCEurLAB/ and in Additional files 1 and 2.

\section{Availability and requirements \\ Project name: DCE@urLAB 1.0 \\ Project home page: http://www.die.upm.es/im/archives/ DCEurLAB/}

Operating system(s): Microsoft Windows 7/Vista/XP

Programming language: IDL

Other requirements: IDL 6.4 or higher, IDL Virtual Machine 6.4 or higher

License: BSD license

\section{Additional files}

Additional file 1: Compressed file (zip format) with executable software, source code, and user manual. Unzip and read the file "/help/DCEurLAB_UserGuide.pdf" for instructions and details.

Additional file 2: Compressed file (zip format) with examples to test and validate the DCE@urLAB application.

\section{Abbreviations}

AIF: Arterial input function; BMS: Bulk magnetic susceptibility; CA: Contrast agent; CPU: Central processing unit; DCE: Dynamic contrast-enhanced; DWI: Diffusion weighted imaging; EES: Extracellular extravascular space; Gd-DTPA: Gadolinium-diethylene-triamine penta-acetic acid; GEM: Genetically

engineered mouse; GUI: Graphical user interface; IAUC: Initial area under curve; IDL: Interactive Data Language; LMA: Levenberg-Marquardt algorithm; MR: Magnetic resonance; MRI: Magnetic resonance imaging; RAM: Random access memory; RR : Reference region; RCE: Relative contrast enhancement; ROI: Region of Interest; TE: Echo time; TR: Repetitition time; TTM: Time to max enhancement.

\section{Competing interests}

The authors declare that they have no competing interests.

\section{Authors' contributions}

JEO, RVS, MJLC and APC participated in the design of the application tool. JEO implemented the software. RVS and APC carried out test of the application and software validation MJLC, CA and AS contributed with data interpretation. CA and AS coordinated the work. All authors contributed with the know-how in biomedical imaging, helped to draft the manuscript and read and approved the final version.

\section{Acknowledgements}

This work was partially supported by Spain's Ministry of Science \& Innovation through CDTI-CENIT (AMIT project), INNPACTO (PRECISION \& XIORT projects), PHENOIMA (SAF 2008-0332), MARESCAN (SAF 2011-23870), projects TEC201021619-C04-03 \& TEC2011-28972-C02-02; Comunidad de Madrid (ARTEMIS S2009/DPI-1802), and IMAFEN (2008-2009), PROGLIO (2010-2011) and PROGLIO2 (2012-2013), intramural projects of CIBER-BBN, with contribution from European Regional Development Funds (FEDER). CIBER-BBN is an initiative funded by the VI National R\&D\&i Plan 2008-2011, Iniciativa Ingenio 2010, Consolider Program, CIBER Actions and financed by the Instituto de Salud Carlos III with assistance from the European Regional Development Fund. 


\section{Author details}

${ }^{1}$ CIBER de Bioingeniería, Biomateriales y Nanomedicina (CIBER-BBN), 50018 Zaragoza, Spain. ${ }^{2}$ Biomedical Image Technologies Group, Departamento de Ingeniería Electrónica, Universidad Politécnica de Madrid, 28040 Madrid, Spain. ${ }^{3}$ Department of Maternal-Fetal Medicine (ICGON), Fetal and Perinatal Medicine Research Group (IDIBAPS), Hospital Clínic, Universitat de Barcelona, Sabino de Arana 1- Helios III, 08028 Barcelona, Spain. ${ }^{4}$ Departament de Bioquímica i Biologia Molecular, Unitat de Biociències, Universitat Autònoma de Barcelona, Edifici Cs, Campus UAB, 08193 Cerdanyola del Vallès, Spain. ${ }^{5}$ Institut de Biotecnologia i de Biomedicina Vicent Villar Palasí (IBB), Universitat Autònoma de Barcelona, Edifici Cs, Campus UAB, 08193 Cerdanyola del Vallès, Spain

Received: 18 July 2013 Accepted: 28 October 2013

Published: 4 November 2013

\section{References}

1. Leach MO, Brindle KM, Evelhoch JL, Griffiths JR, Horsman MR, Jackson A, Jayson GC, Judson IR, Knopp MV, Maxwell RJ, McIntyre D, Padhani AR, Price P, Rathbone R, Rustin GJ, Tofts PS, Tozer GM, Vennart W, Waterton JC, Williams SR, Workmanw P: The assessment of antiangiogenic and antivascular therapies in early-stage clinical trials using magnetic resonance imaging: issues and recommendations. Brit J Cancer 2005 92(9):1599-1610.

2. Yankeelov TE, Gore JC: Dynamic contrast enhanced magnetic resonance imaging in oncology: theory, data acquisition, analysis, and examples. Curr Med Imaging Rev 2007, 3(2):91-107.

3. Choyke PL, Dwyer AJ, Knopp MV: Functional tumor imaging with dynamic contrast-enhanced magnetic resonance imaging. J Magn Reson Imaging 2003, 17(5):509-520.

4. Collins DJ, Padhani AR: Dynamic magnetic resonance imaging of tumor perfusion. IEEE Eng Med Biol 2004, 23(5):65-83.

5. Tofts PS: Modeling tracer kinetics in dynamic Gd-DTPA MR imaging. J Magn Reson Imaging 1997, 7:91-101.

6. Kiessling F, Farhan N, Lichy MP, Vosseler S, Heilmann M, Krix M, Bohlen P, Miller DW, Mueller MM, Semmler W, Fusenig NE, Delorme S: Dynamic contrast-enhanced magnetic resonance imaging rapidly indicates vessel regression in human squamous cell carcinomas grown in nude mice caused by VEGF receptor 2 blockade with DC101. Neoplasia 2004, 6(3):213-223.

7. Pike MM, Stoops CN, Langford CP, Akella NS, Nabors LB, Gillespie GY: High-resolution longitudinal assessment of flow and permeability in mouse glioma vasculature: sequential small molecule and SPIO dynamic contrast agent MRI. Magn Reson Med 2009, 61(3):615-625.

8. Tofts PS, Kermode AG: Measurement of the blood-brain barrier permeability and leakage space using dynamic MR imaging. 1. fundamental concepts. Magn Reson Med 1991, 17(2):357-367.

9. Hoffmann U, Brix G, Knopp MV, Hess T, Lorenz WJ: Pharmacokinetic mapping of the breast - a new method for dynamic MR mammography. Magn Reson Med 1995, 33(4):506-514.

10. Larsson HBW, Stubgaard M, Frederiksen JL, Jensen M, Henriksen $O$, Paulson OB: Quantitation of blood-brain-barrier defect by magneticresonance-imaging and gadolinium-DTPA in patients with multiple sclerosis and brain tumors. Magn Reson Med 1990, 16:117-131.

11. Yankeelov TE, Luci JJ, Lepage M, Li R, Debusk L, Lin PC, Price RR, Gore JC: Quantitative pharmacokinetic analysis of DCE-MRI data without an arterial input function: a reference region model. Magn Reson Imaging 2005, 23(4):519-529.

12. Weidensteiner C, Rausch M, McSheehy PMJ, Allegrini PR: Quantitative dynamic contrast-enhanced MRI in tumor-bearing rats and mice with inversion recovery TrueFISP and two contrast agents at 4.7 T. J Magn Reson Imaging 2006, 24(3):646-656.

13. Kim JH, Im GH, Yang J, Choi D, Lee WJ, Lee JH: Quantitative dynamic contrast-enhanced MRI for mouse models using automatic detection of the arterial input function. NMR Biomed 2012, 25(4):674-684.

14. Jensen LR, Huuse EM, Bathen TF, Goa PE, Bofin AM, Pedersen TB, Lundgren S, Gribbestad IS: Assessment of early docetaxel response in an experimental model of human breast cancer using DCE-MRI, ex vivo HR MAS, and in vivo H-1 MRS. NMR Biomed 2010, 23:56-65.
15. Cho HJ, Ackerstaff E, Carlin S, Lupu ME, Wang Y, Rizwan A, O'Donoghue J, Ling CC, Humm JL, Zanzonico PB, Koutcher JA: Noninvasive multimodality imaging of the tumor microenvironment: registered dynamic magnetic resonance imaging and positron emission tomography studies of a preclinical tumor model of tumor hypoxia. Neoplasia 2009, 11(3):247-259

16. Muruganandham M, Lupu M, Dyke JP, Matei C, Linn M, Packman K, Kolinsky K, Higgins B, Koutcher JA: Preclinical evaluation of tumor microvascular response to a novel antiangiogenic/antitumor agent RO0281501 by dynamic contrast-enhanced MRI at 1.5 T. Mol Cancer Ther 2006, 5(8):1950-1957.

17. Yang XY, Liang JC, Heverhagen JT, Jia G, Schmalbrock P, Sammet S, Koch $R$, Knopp MV: Improving the pharmacokinetic parameter measurement in dynamic contrast-enhanced MRI by use of the arterial input function: theory and clinical application. Magn Reson Med 2008, 59(6):1448-1456.

18. Stefanovski D, Moate PJ, Boston RC: WinSAAM: a windows-based compartmental modeling system. Metabolism 2003, 52(9):1153-1166.

19. JPKD 3.0, Java PK for Desktop. http://pkpd.kmu.edu.tw/jpkd/

20. Barrett PHR, Bell BM, Cobelli C, Golde H, Schumitzky A, Vicini P, Foster DM: SAAM II: simulation, analysis, and modeling software for tracer and pharmacokinetic studies. Metabolism 1998, 47(4):484-492.

21. Rausch M, Stoeckli M: BioMap. http://www.maldi-msi.org/.

22. Cetin O: An analysis tool to calculate permeability based on the patlak method. J Med Syst 2012, 36(3):1317-1326.

23. Daniel P: Barboriak Laboratory, Duke University School of Medicine: Toppcat, T1-weighted Perfusion Imaging Parameter Calculation Toolkit. http://dblab.duhs.duke.edu/modules/dblabs_topcat/.

24. Whitcher B, Schmid VJ: Quantitative analysis of dynamic contrast-enhanced and diffusion-weighted magnetic resonance imaging for oncology in R. J Stat Softw 2011, 44(5):1-29.

25. Ferl GZ: DATforDCEMRI: an $\mathbf{R}$ package for deconvolution analysis and visualization of DCE-MRI data. J Stat Softw 2011, 44(3):1-18

26. Keyzer FD, Vandecaveye V, Thoeny H, Chen F, Ni Y, Marchal G, Hermans R, Nuyts S, Landuyt W, Bosmans H: Dynamic Contrast-enhanced and diffusion-weighted MRI for early detection of tumoral changes in single-dose and fractionated radiotherapy: evaluation in a rat rhabdomyosarcoma model. Eur Radiol 2009, 19(11):2663-2671.

27. Wang HJ, Li JJ, Chen F, Keyzer FD, Yu J, Feng YB, Nuyts J, Marchal G, Ni YC: Morphological, functional and metabolic imaging biomarkers: assessment of vascular-disrupting effect on rodent liver tumours. Eur Radiol 2010, 20(8):2013-2026.

28. Patlak CS, Blasberg RG, Fenstermacher JD: Graphical evaluation of blood-to-brain transfer constants from multiple-time uptake data. J Cerebr Blood F Met 1983, 3:1-7.

29. U. S. National Institutes of Health Bethesda, MD: ImageJ, Image Processing and Analysis in Java. http://imagej.nih.gov/ij/

30. R Development Core Team: R: A language and Environment for Statistical Computing. Vienna: R Foundation for Statistical Computing; 2011. http://www.R-project.org/.

31. Orton MR, d'Arcy JA, Walker-Samuel S, Hawkes DJ, Atkinson D, Collins DJ, Leach MO: Computationally efficient vascular input function models for quantitative kinetic modelling using DCE-MRI. Phys Med Biol 2008, 53(5):1225-1239.

32. Ferl GZ, Xu L, Friesenhahn M, Bernstein $\sqcup$, Barboriak DP, Port RE: An automated method for nonparametric kinetic analysis of clinical DCE-MRI data: application to glioblastoma treated with bevacizumab. Magn Reson Med 2010, 63(5):1366-1375.

33. Bradley DP, Tessier JJ, Lacey T, Scotta M, Jurgensmeier JM, Odedra R, Mills J, Kilburn L, Wedge SR: Examining the acute effects of cediranib (RECENTIN, AZD2171) treatment in tumor models: a dynamic contrast-enhanced MRI study using gadopentate. Magn Reson Imaging 2009, 27(3):377-384.

34. Loveless ME, Lawson D, Collins M, Nadella MVP, Reimer C, Huszar D, Halliday J, Waterton JC, Gore JC, Yankeelov TE: Comparisons of the efficacy of a Jak1/2 Inhibitor (AZD1480) with a VEGF signaling inhibitor (cediranib) and sham treatments in mouse tumors using DCE-MRI, DW-MRI, and histology. Neoplasia 2012, 14:54-64.

35. Kim H, Folks KD, Guo LL, Stockard CR, Fineberg NS, Grizzle WE, George JF, Buchsbaum DJ, Morgan DE, Zinn KR: DCE-MRI detects early vascular 
response in breast tumor xenografts following anti-DR5 therapy. Mol Imaging Biol 2011, 13:94-103.

36. Yankeelov TE, DeBusk LM, Billheimer DD, Luci JJ, Lin PC, Price RR, Gore JC: Repeatability of a reference region model for analysis of murine DCE-MRI data at 7T. J Magn Reson Imaging 2006, 24(5):1140-1147.

37. Kato $Y$, Okollie B, Artemov D: Noninvasive H-1/C-13 magnetic resonance spectroscopic imaging of the intratumoral distribution of temozolomide. Magn Reson Med 2006, 55(4):755-761.

38. Artemov D, Solaiyappan M, Bhujwalla ZM: Magnetic resonance pharmacoangiography to detect and predict chemotherapy delivery to solid tumors. Cancer Res 2001, 61(7):3039-3044.

39. Luo YP, Jiang F, Cole TB, Hradil VP, Reuter D, Chakravartty A, Albert DH, Davidsen SK, Cox BF, McKeegan EM, Fox GB: A novel multi-targeted tyrosine kinase inhibitor, linifanib (ABT-869), produces functional and structural changes in tumor vasculature in an orthotopic rat glioma model. Cancer Chemoth Pharm 2012, 69(4):911-921.

40. Port RE, Knopp MV, Hoffmann U, Milker-Zabel S, Brix G: Multicompartment analysis of gadolinium chelate kinetics: blood-tissue exchange in mammary tumors as monitored by dynamic MR imaging. J Magn Reson Imaging 1999, 10(3):233-241.

41. Kety SS: The theory and applications of the exchange of inert gas at the lungs and tissues. Pharmacol Rev 1951, 3:1-41.

42. Tofts PS, Berkowitz B, Schnall MD: Quantitative analysis of dynamic Gd-DTPA enhancement in breast tumors using a permeability model. Magn Reson Med 1995, 33(4):564-568.

43. Sourbron SP, Buckley DL: On the scope and interpretation of the Tofts models for DCE-MRI. Magn Reson Med 2011, 66(3):735-745.

44. Brix G, Semmler W, Port R, Schad LR, Layer G, Lorenz WJ: Pharmacokinetic parameters in CNS Gd-DTPA enhanced MR imaging. J Cerebr Blood F Met 1991, 15(4):621-628.

45. Haase A, Frahm J, Matthaei D, Hanicke W, Merboldt KD: Flash imaging rapid NMR imaging using low flip-angle pulses. J Magn Reson 1986, 67(2):258-266.

46. Li X, Rooney WD, Varallyay CG, Gahramanov S, Muldoon LL, Goodman JA, Tagge IJ, Selzer AH, Pike MM, Neuwelt EA, Springer CS:

Dynamic-contrast-enhanced-MRI with extravasating contrast reagent: rat cerebral glioma blood volume determination. J Magn Reson 2010, 206(2):190-199.

47. Markwardt CB: Non-linear least squares fitting in IDL with MPFIT. In CXVIII, Volume 411. Edited by Bohlender D, Dowler P, Durand D. Quebec: Astronomical Society of the Pacific; 2009:251-254.

48. Marquardt DW: An algorithm for least-squares estimation of nonlinear parameters. J Soc Ind App/ Math 1963, 11(2):431-441.

49. Ahearn TS, Staff RT, Redpath TW, Semple SIK: The use of the Levenberg-Marquardt curve-fitting algorithm in pharmacokinetic modelling of DCE-MRI data. Phys Med Biol 2005, 50(9):N85-N92.

50. Parker GJM, Buckley DL: Tracer kinetic modelling for T1-weighted DCE-MRI. In Dynamic Contrast-Enhanced Magnetic Resonance Imaging in Oncology, Medical Radiology: Diagnostic, Imaging and Radiation Oncology. Edited by Jackson A, Buckley DL, Parker GJM. Berlin: Springer-Verlag; 2003:81-92.

51. Barboriak DP, MacFall JR, Viglianti BL, Dewhirst MW: Comparison of three physiologically-based pharmacokinetic models for the prediction of contrast agent distribution measured by dynamic MR imaging. J Magn Reson Imaging 2008, 27(6):1388-1398.

52. Raymond GM, Butterworth E, Bassingthwaighte JB: JSIM: free software package for teaching physiological modeling and research. FASEB $J$ 2003, 17(4):A390-A390.

53. Weiss WA, Burns MJ, Hackett C, Aldape K, Hill JR, Kuriyama H, Kuriyama N, Milshteyn N, Roberts T, Wendland MF, DePinho R, Israel MA: Genetic determinants of malignancy in a mouse model for oligodendroglioma. Cancer Res 2003, 63(7):1589-1595.

54. Delgado-Goni T, Martin-Sitjar J, Simoes RV, Acosta M, Lope-Piedrafita S, Arús C: Dimethyl sulfoxide (DMSO) as a potential contrast agent for brain tumors. NMR Biomed 2013, 26(2):173-184.

55. Cha S, Johnson G, Wadghiri YZ, Jin O, Babb J, Zagzag D, Turnbull DH: Dynamic, contrast-enhanced perfusion MRI in mouse gliomas: correlation with histopathology. Magn Reson Med 2003, 49(5):848-855.
56. Simoes RV, García-Martín ML, Cerdán S, Arús C: Perturbation of mouse glioma MRS pattern by induced acute hyperglycemia. NMR Biomed 2008, 21(3):251-264.

57. Stoyanova R, Huang K, Sandler K, Cho H, Carlin S, Zanzonico PB, Koutcher JA, Ackerstaff E: Mapping tumor hypoxia in vivo using pattern recognition of dynamic contrast-enhanced MRI data. Trans Oncol 2012, 5(6):437-447.

doi:10.1186/1471-2105-14-316

Cite this article as: Ortuño et al.: DCE@urLAB: a dynamic contrast-enhanced MRI pharmacokinetic analysis tool for preclinical data. BMC Bioinformatics 2013 14:316.

\section{Submit your next manuscript to BioMed Central and take full advantage of:}

- Convenient online submission

- Thorough peer review

- No space constraints or color figure charges

- Immediate publication on acceptance

- Inclusion in PubMed, CAS, Scopus and Google Scholar

- Research which is freely available for redistribution
Biomed Central 